\title{
Field Squeezing in a Quantum-Dot Molecule Jaynes-Cummings Model
}

\author{
Xu Chu, ${ }^{1}$ Xiaodong Ma, ${ }^{2}$ Ying Wang, ${ }^{1,3}$ Qingchun Zhou, ${ }^{1}$ and Yu Zhou ${ }^{1}$ \\ ${ }^{1}$ School of Mathematics and Physics, Jiangsu University of Science and Technology, Jiangsu 212003, China \\ ${ }^{2}$ Department of Physics and Electronic Engineering, Xinjiang Normal University, Xinjiang 830054, China \\ ${ }^{3}$ Benjamin M. Statler College of Engineering and Mineral Resources, West Virginia University, Morgantown, WV 26505, USA
}

Correspondence should be addressed to Yu Zhou; yzhou@just.edu.cn

Received 9 April 2014; Accepted 12 June 2014; Published 3 July 2014

Academic Editor: Kim Fook Lee

Copyright (C) $2014 \mathrm{Xu}$ Chu et al. This is an open access article distributed under the Creative Commons Attribution License, which permits unrestricted use, distribution, and reproduction in any medium, provided the original work is properly cited.

\begin{abstract}
We investigate the field squeezing in a system composed of an initial coherent field interacting with two quantum dots coupled by electron tunneling. An approximate quantum-dot molecule Jaynes-Cummings model describing the system is given, and the effects of physical quantities, such as the temperature, phonon-electron interaction, mean photon number, field detuning, and tunneling-level detuning, are discussed in detail.
\end{abstract}

\section{Introduction}

Thanks to the rapid progress of nanotechnologies, the exploration of the quantum-dot system, a mesoscopic synthetic material with a quantum confinement configuration, has provided a versatile tool for the simulation of many interesting phenomena in condensed matter physics $[1,2]$, among which, the quantum-dot molecule (QDM), formed from an asymmetric double quantum-dot system coupled by tunneling, has attracted much attention recently. New trends in nanotechnology even enable us to manipulate this kind of QDM using the external controlment, that is, electric field or optical field.

The interaction between atoms and field, an intriguing topic in modern quantum optics, yields many novel properties of the optical system. Based on the study of JaynesCummings model, a fully quantum mechanical model and one of the few exactly solvable models that describes an atom in an external field, many studies have been made including the generalization of the model, such as applying the initial conditions [3], considering the dissipation and damping [4], multilevel of atoms, and multimode field [5, 6]. Many nonclassical effects have been investigated within the framework of this model, like Rabi oscillations, collapserevival phenomenon, sub-Poissonian photon statistics, and squeezed state of the radiation field $[7,8]$. Specifically, the squeezed state of light, a pure quantum effect without classical parallelism, has attracted much attention in these decades [9]; it can be widely used in the exploration of gravitational wave [10, 11], nonlinear optics [12], quantum information [13], quantum communication [14], and even precision metrology [15].

For its artificial nature and properties similar to natural atoms and molecules, the QDM, an ideal candidate for the mimic of atoms interacting with external fields, has been studied extensively, because its levels could be controlled conveniently and tuned easily [16]. However, the QDM is often surrounded by the solid matrix which means that the electrons are inevitably coupled with phonons and it hints that the electron-phonon interaction cannot be neglected during the tunneling. Therefore the zero-temperature treatment is not always valid and the temperature of the environment has a significant role [17]. Although the population of all states has been analyzed, the squeezing effect of the QDM has not been investigated. In this paper we consider the squeezing of a single-mode field interacting with a QDM and study analytically and numerically how the squeezing depends on various tunable parameters of the system.

This paper is arranged as follows; the next section gives the model of the external field interacting with a QDM and 
the equations describing the system are given; in Section 3 the squeezing of the optical field is considered from different perspectives while Section 4 gives the discussion and summary.

\section{The Model}

Consider a pulsed laser field interacting with a QDM with the configuration that state $|0\rangle$ is the ground state without excitation while states $|1\rangle$ and $|2\rangle$ are excited states representing an electron in the left dot and one hole in the left dot [16]. For the sake of the far off-resonance of the valence band levels of two dots, other states are hardly coupled with these states; hence they are neglected here for simplicity without the loss of generality. The single-mode field with frequency $\omega$ couples the states $|0\rangle$ and $|1\rangle$ with detuning $\delta$; states $|0\rangle$ and $|2\rangle$ are uncoupled in the time scale we consider here; quantum electronic tunneling $T_{e}$ between states $|1\rangle$ and $|2\rangle$ can be controlled by voltage.

Because of the low-enough temperature considered here, most of the electron-phonon interaction is the result of the deformation of the potential while the coupling constant $g_{k}$ of this interaction is determined by the quantum-dot material and lattice configuration. Using the assumption that other factors causing the interaction are negligible, hence all the phonon-electron interactions can be treated as caused by the potential deformation [18]. So it is reasonable to apply the Einstein model with all the phonons having the same frequency $\omega_{k}=\omega_{0}$. Under the dipole approximation and the rotating-wave approximation, the Hamiltonian of the system in the interaction picture reads $(\hbar=1)$ [19]:

$$
\begin{aligned}
H_{I}= & T_{e}\left(R_{21} e^{-i \omega_{12} t}+R_{21} e^{i \omega_{12} t}\right) e^{-S} \\
& +\lambda\left(\hat{a}^{\dagger} R_{01} e^{-i(\delta-\Delta) t}+\widehat{a} R_{10} e^{i(\delta-\Delta) t}\right) e^{-S / 2},
\end{aligned}
$$

where $\Delta=\sum_{k} g_{k}^{2} /\left(4 \omega_{k}\right)$ and detuning $\delta=\omega_{10}-\omega$, $\omega_{12}=\omega_{1}-\omega_{2}$. The item representing the phonon effects, which has the expression $S=2 \sum_{k}(N+1 / 2)\left(g_{k} / 2 \omega_{k}\right)^{2}=$ $2(N+1 / 2) \sum_{k}\left(g_{k}^{2} / 4 \omega_{k}^{2}\right)=2(N+1 / 2) G$, where $G$ is HuangRhys factor expressed as $\sum_{k}\left(g_{k}^{2} / 4 \omega_{k}^{2}\right)$ [20], corresponds to the electron-phonon interaction with temperature-dependent phonon population $N=1 /\left(e^{\omega_{k} / T}-1\right)$. In Hamiltonian (1), $R_{i j}=|i\rangle\langle j|(i, j=0,1,2)$ corresponds to the transition operators of the QDM, $T_{e}$ is tunneling matrix element between the left and right dots, $\lambda$ is the field-QDM coupling constant, and $\hat{a}^{\dagger}(\widehat{a})$ is the creation (annihilation) operator of the optical field satisfying $\left[\widehat{a}, \widehat{a}^{\dagger}\right]=1$. In the model, both $\omega_{12}$ and $T_{e}$ can be manipulated by an external voltage.

We suppose that initially the QDM is in the ground state $|0\rangle$ and the system is described as

$$
|\psi(0)\rangle=\left|\psi_{A}(0)\right\rangle \otimes\left|\psi_{F}(0)\right\rangle=\sum_{n=0}^{\infty} F_{n}|0, n\rangle,
$$

where $\left|\psi_{F}(0)\right\rangle$ is the arbitrary optical field, and then the state vector of the system at time $t$ takes the form

$$
|\psi(t)\rangle=\sum_{n}\left[c_{n}(t)|2, n\rangle+b_{n}(t)|1, n\rangle+a_{n+1}(t)|0, n+1\rangle\right] ;
$$

hence the substitution of state vector (3) into Schrödinger's equation yields a set of differential equations describing the expanding coefficients as

$$
\begin{aligned}
& i\left(\begin{array}{c}
\dot{a}_{n+1} \\
\dot{b}_{n} \\
\dot{c}_{n}
\end{array}\right) \\
& =\left(\begin{array}{ccc}
0 & \lambda \sqrt{n+1} e^{-i(\delta-\Delta) t} e^{-S / 2} & 0 \\
\lambda \sqrt{n+1} e^{i(\delta-\Delta) t} e^{-S / 2} & 0 & T_{e} e^{i \omega_{12} t} e^{-S} \\
0 & T_{e} e^{-i \omega_{12} t} e^{-S} & 0
\end{array}\right) \\
& \times\left(\begin{array}{c}
a_{n+1} \\
b_{n} \\
c_{n}
\end{array}\right) .
\end{aligned}
$$

The combination of the above coupled equations leads to a set of decoupled equations and their solutions could be obtained with the initial conditions $a_{n+1}(0)=F_{n+1}$ and $b_{n}(0)=c_{n}(0)=$ 0 .

Analytically, the solutions can be directly given when phonon effect is not included for $\omega_{12}=\delta, \Delta=0, \delta=\Delta$, and $\omega_{12}=0$; or the external optical field is in resonance with the zero-phonon line while the state $|1\rangle$ is in resonance with state $|2\rangle$ which indicates that the coherent population oscillations can persist, similar to the case without phonon effect, and expect that the expression in the Rabi like oscillating frequency and the interdot tunneling rate is renormalized by the exponents of $-S / 2$ and $-S$ [17]. We can see that electronphonon coupling can make a significant contribution to the light-QDM interaction which can make the oscillation of the population of the state more drastic than that without the phonon effect [19].

\section{Squeezing of the System}

We introduce two orthogonal quadrature amplitude operators $\widehat{X}_{1}=(1 / 2)\left(\widehat{a}+\widehat{a}^{\dagger}\right)$ and $\widehat{X}_{2}=(1 / 2 i)\left(\widehat{a}-\widehat{a}^{\dagger}\right)$ satisfying the commutation relation $\left[\widehat{X}_{1}, \widehat{X}_{2}\right]=i / 2$ which lead to

$$
\left(\Delta \widehat{X}_{1}\right)^{2}\left(\Delta \widehat{X}_{2}\right)^{2} \geq \frac{1}{16}
$$

according to Heisenberg's uncertainty principle, with $\left(\Delta \widehat{X}_{i}\right)^{2}=\left\langle\widehat{X}_{i}^{2}\right\rangle-\left\langle\widehat{X}_{i}\right\rangle^{2}(i=1,2)$. The state of the field is said to be squeezed when one of the amplitudes $\widehat{X}_{i}$ satisfies the 
relation $Q_{i}=\left(\Delta \widehat{X}_{i}\right)^{2}-1 / 4 \leq 0(i=1,2)$. According to (3) we express the squeezing parameters for the two amplitudes as

$$
\begin{aligned}
& Q_{1}=\frac{1}{4} \sum_{n} \sqrt{(n+1)(n+2)} \\
& \times\left(c_{n}^{*} c_{n+2}+b_{n}^{*} b_{n+2}+a_{n}^{*} a_{n+2}\right. \\
&\left.+c_{n+2}^{*} c_{n}+b_{n+2}^{*} b_{n}+a_{n+2}^{*} a_{n}\right) \\
&+ \frac{1}{4} \sum_{n}(2 n+1)\left(c_{n}^{*} c_{n}+b_{n}^{*} b_{n}+a_{n}^{*} a_{n}\right) \\
&-\frac{1}{4}\left[\sum_{n} \sqrt{n+1}\right. \times\left(c_{n}^{*} c_{n+1}+b_{n}^{*} b_{n+1}+a_{n}^{*} a_{n+1}\right. \\
&\left.\left.+c_{n+1}^{*} c_{n}+b_{n+1}^{*} b_{n}+a_{n+1}^{*} a_{n}\right)\right]^{2}-\frac{1}{4}, \\
& Q_{2}=-\frac{1}{4} \sum_{n} \sqrt{(n+1)(n+2)} \\
& \times\left(c_{n}^{*} c_{n+2}+b_{n}^{*} b_{n+2}+a_{n}^{*} a_{n+2}+c_{n+2}^{*} c_{n}\right. \\
&\left.+b_{n+2}^{*} b_{n}+a_{n+2}^{*} a_{n}\right) \\
&+\frac{1}{4} \sum_{n}(2 n+1)\left(c_{n}^{*} c_{n}+b_{n}^{*} b_{n}+a_{n}^{*} a_{n}\right)-\frac{1}{4} .
\end{aligned}
$$

Making the assumption that the optical field is initially in a monochromatic coherent state $F_{n}=e^{-\bar{n} / 2} \bar{n}^{n / 2} e^{i n \beta} / \sqrt{n !}$, where the mean photon number of the coherent fields $\bar{n}=|\alpha|^{2}$ and $\beta$ is the phase, $\beta=0$ is chosen in the following.

In order to investigate the squeezing in detail, we consider several factors that affect squeezing behaviors. First the temperature effect of the system is discussed, where $G=0.01$ represents the electron-phonon interaction and $\Delta=\delta=$ $0.05 \omega, \omega_{12}=0, \lambda=0.2 \omega$, and $T_{e}=0.2 \omega$; we choose different values of temperature $T$ which directly modify the phonon population. In Figure 1 we show that, with the evolution of dimensionless time $\omega t$, the curves change significantly with the increase of temperature $T$. In the zero-temperature case (Figure 1(a)), the envelope of oscillation is sinusoidal but with a significant damping. In the time scale shown in the figure, there are more than three time regions where the system reaches squeezing for $Q_{1}$; however, the number of time ranges for the squeezing of $Q_{2}$ is even more but the amplitude of $Q_{2}$ is smaller than that of $Q_{1}$. We also notice that the oscillation frequencies of the squeezing parameters at higher temperatures are smaller than at lower ones. It is apparent that, for higher temperatures, $T=50 \omega_{0}$ and $T=100 \omega_{0}$, chances for the squeezing decrease for both $Q_{1}$ and $Q_{2}$. The physical explanation is obvious: the quantum properties are weakened for the increase of temperature; being a typical quantum phenomenon, squeezing is strongly suppressed by higher temperature.

Now we set a fixed temperature $T=10 \omega_{0}$ (use this temperature hereafter) and assume the detuning $\delta=\omega_{12}=$ 0 , electron-phonon interaction factor $G=0.01$, interdot

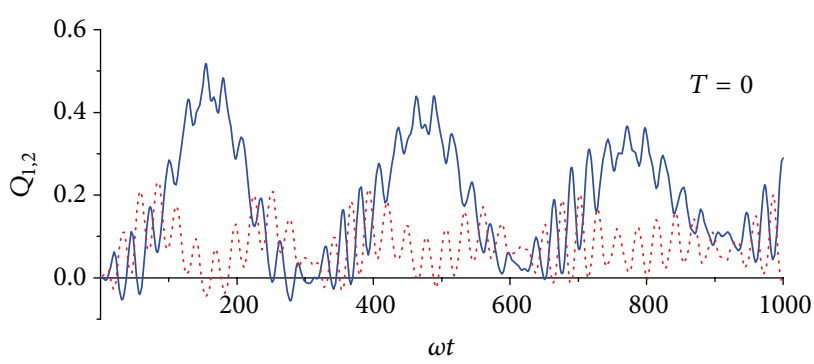

(a)

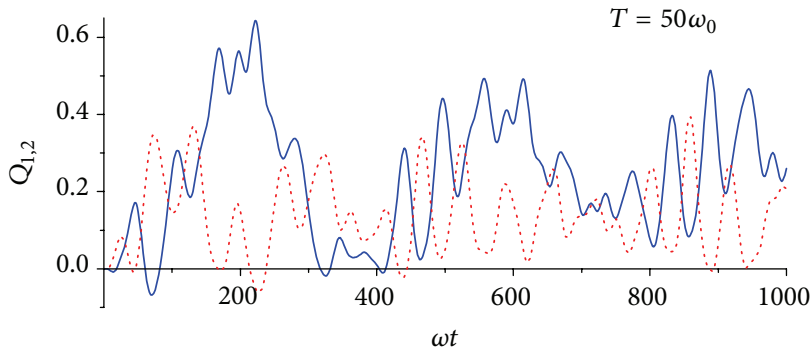

(b)

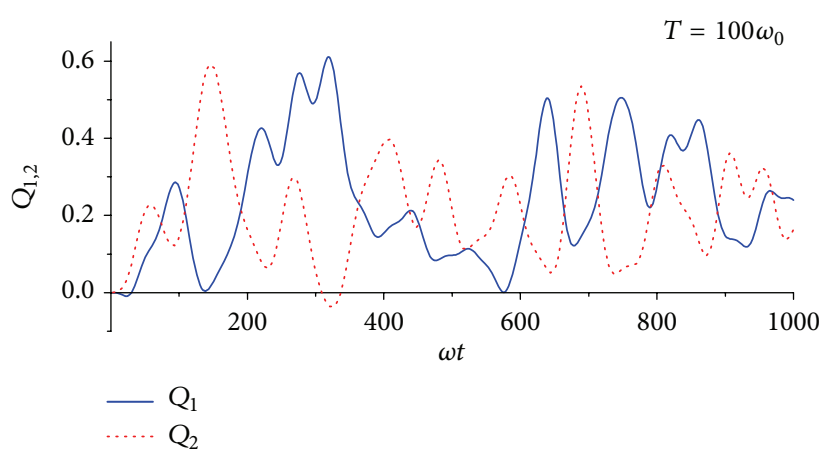

(c)

Figure 1: (Color online) $Q_{1,2}$ versus dimensionless time $\omega t$ at different temperature when $\Delta=\delta=0.05 \omega, \omega_{12}=0, G=0.01$, $T_{e}=0.2 \omega$, and $\lambda=0.2 \omega$ are considered.

tunneling $T_{e}=0.2 \omega$, and field-QDM coupling $\lambda=0.1 \omega$; then Figure 2 shows that when $\Delta$ varies from $0.01 \omega$ to $0.05 \omega$ the squeezing of the states significantly decreases, indicated by both $Q_{1}$ and $Q_{2}$ curves. The biggest squeezing appears for the smallest $\Delta$; we find that the maximum squeezing reaches $24 \%(12 \%)$ for $Q_{1}\left(Q_{2}\right)$ when $\Delta=0.01 \omega$ while the squeezing reduces to $2 \%(2.4 \%)$ when $\Delta$ increases to $0.05 \omega$. Also we find that, with the growth of $\Delta$, the periodicity vanishes for long time scale (not shown in the figure) which means that the gradual enhancement phonon effect will lead to the irregular oscillations of the squeezing curves. The biggest squeezing happens around $\omega t=40(20)$ for $Q_{1}\left(Q_{2}\right)$ at small $\Delta$ and, after the time around $\omega t=40$, the system will not go back to the squeezed state any more.

It is interesting to speculate how the squeezing changes with detuning $\omega_{12}$ at fixed temperature and its timedependent behaviors. In this case, we choose $\Delta=0$ and $\omega_{12}$ is ranging from $0.01 \omega$ to $0.1 \omega ; T_{e}=0.2 \omega, T=10 \omega_{0}$, and $\lambda=0.1 \omega$ are fixed as previously indicated. Noticing from 


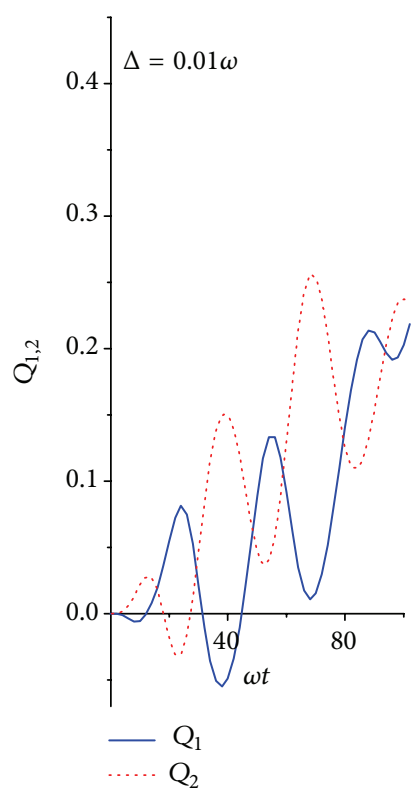

(a)

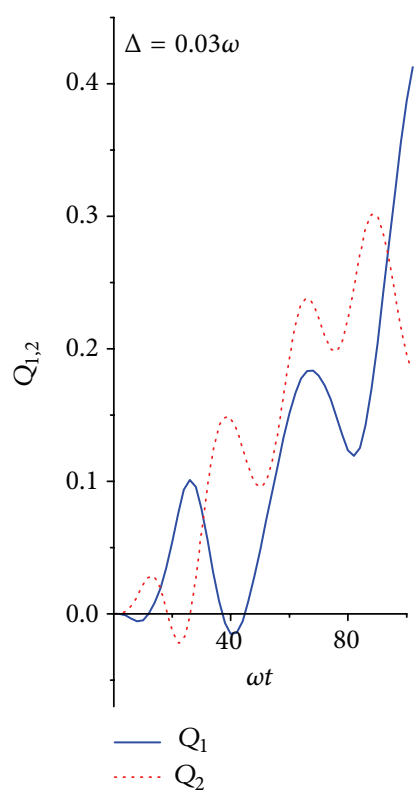

(b)

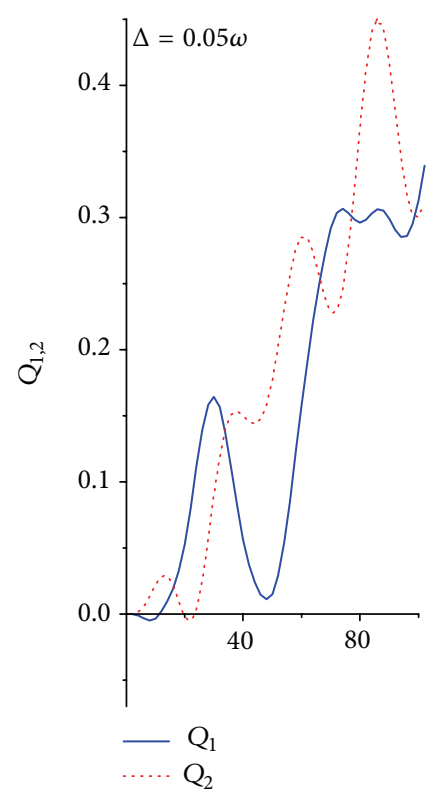

(c)

FIGURE 2: (Color online) $Q_{1,2}$ versus dimensionless time $\omega t$ for different $\Delta$ when $T=10 \omega_{0}, \delta=\omega_{12}=0, G=0.01, T_{e}=0.2 \omega$, and $\lambda=0.1 \omega$ are chosen.

Figure 3 clearly that squeezing decreases with the increase of $\omega_{12}$, the biggest squeezing for $Q_{1}\left(Q_{2}\right)$ in $\omega_{12}=0.01 \omega$ is $24 \%(12 \%)$ while it decreases to $12 \%(8 \%)$ when $\omega_{12}=0.03 \omega$, and notice, from Figure 3(d), however, there is almost no squeezing when $\omega_{12}=0.1 \omega$ right after the system evolves. The curves in the figure also show that the squeezing of $Q_{1}$ is stronger than $Q_{2}$ regardless of $\omega_{12}$ being small or big.

Previously we suppose that one or two parameters of $\delta$, $\omega_{12}$, and $\Delta$ are zero and get some properties of the squeezing in different regimes. Now, to study the general case that both $\delta$ and $\omega_{12}$ are nonzero and effect of $\Delta$ is included, we show the squeezing parameters in Figure 4 with three sets of values for $\omega_{12}$ less than, equal to, and greater than $\delta$ separately. From the figure we find that when $\omega_{12}<\delta$, the squeezing of $Q_{2}$ is greater than $Q_{1}$ and the biggest squeezing for $Q_{1}\left(Q_{2}\right)$ is about $4 \%$ (8\%); then with the continuous increasing of ratio $\omega_{12} / \delta$ we find that squeezing for $Q_{2}$ is suppressed and eventually vanishes when $\omega_{12}=3 \delta$. Notice that there are two regions for $X_{1}$ being squeezed when $\omega_{12}<\delta$ while the number of regions of squeezing reduces to one if the ratio of $\omega_{12} / \delta$ increases; besides, the squeezing depth and duration of the first region nearly remain unchanged.

Despite the cases that the mean photon number $\bar{n}=1$ discussed above, it is significant to consider the effect of mean photon number $\bar{n}$ on the squeezing; we choose $\Delta=\delta$ and states $|1\rangle$ and $|2\rangle$ are in resonance. When $\bar{n}=1$, we find that the oscillation for $Q_{1,2}$ is periodical with damping in the time range and the squeezing parameter evolves regularly; the biggest squeezing for $Q_{1}$ is around $25 \%$ happening at $\omega t=38$ while around $21 \%$ at $\omega t=170$ for $Q_{2}$. With the time evolution, the field can still be squeezed in the long time scale. When it comes to the bigger $\bar{n}$ cases, we find that, after the system reaches squeezing, the curves almost linearly increase monotonically, which correspond to the so-called Cummings collapse region [21, 22]. We find that the biggest squeezing will be higher than that in $\bar{n}=1$ case. If we want to obtain the biggest squeezing, the laser-QDM interaction time will be longer for larger $\bar{n}$.

The discussion and results above are reliable and they can be implemented in labs. Now we consider the parameters that are used in InAs self-assembled QDM under coherent laser excitation and find that the squeezing for weak and strong tunneling is $6.4 \%$ and $22 \%$, respectively, where we choose $\omega=$ $1.6 \mathrm{eV}, \lambda=1 \times 10^{-3} \mathrm{eV}, \omega_{12}=0.1 \times 10^{-3} \mathrm{eV}, \delta=0.1 \times 10^{-3} \mathrm{eV}$, $\Delta=0, T_{e}=0.1 \times 10^{-3} \mathrm{eV}$ (weak tunnel), or $5 \times 10^{-3} \mathrm{eV}$ (strong tunnel) [23-28]; we find that the squeezing in weak tunneling is greater than that in strong tunneling case which shows that the coupling of the laser field and the QDM is equivalently weakened.

\section{Conclusion}

In this paper we have investigated the field squeezing of the quantum-dot molecule Jaynes-Cummings model. Using the appropriate approximation we give the Hamiltonian of the system describing the field-QDM interaction and solve the related equations afterwards. By analytical and numerical processes, we show influence of the various parameters of the system on the squeezing. We show that the squeezing generally decreases with time. It is shown that, because of the quantum nature being suppressed, the amount of squeezing becomes smaller as temperature rises, and the periodicity of the squeezing parameter curves is destroyed. 


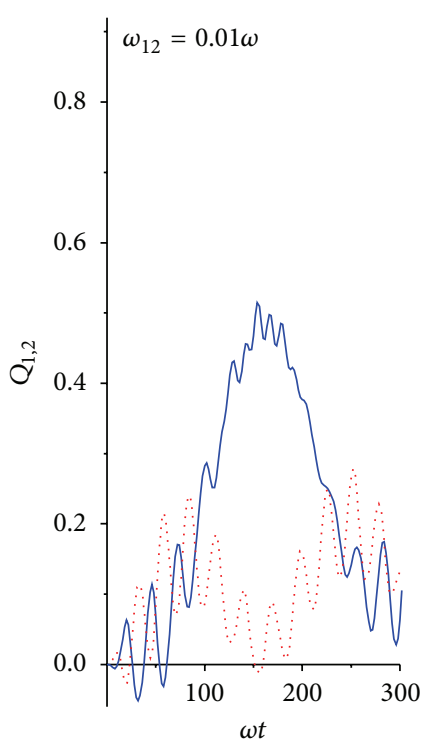

(a)

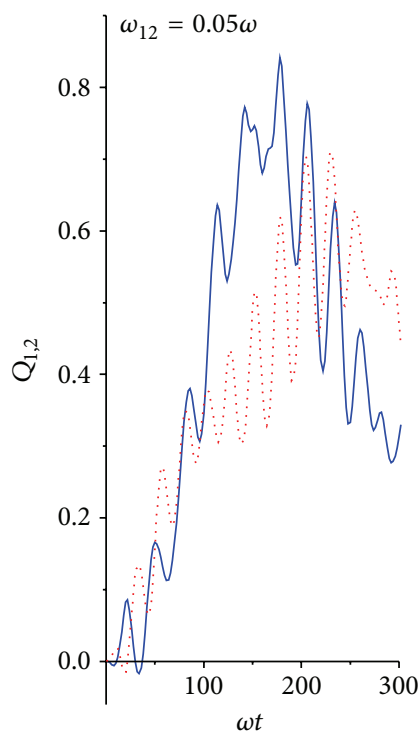

$-Q_{1}$

(c)

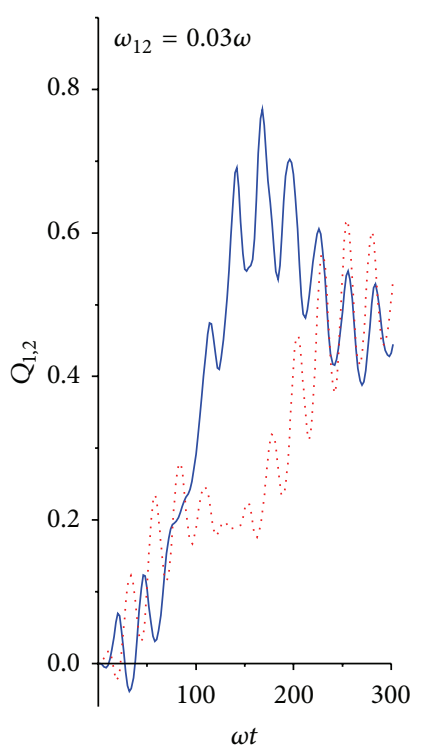

(b)

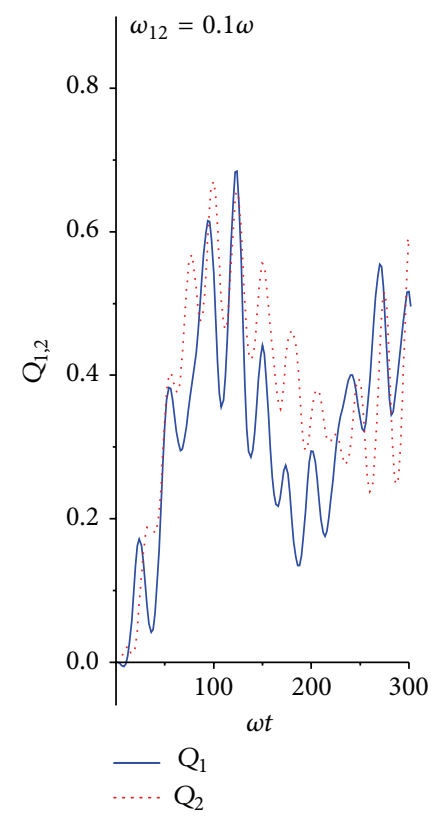

(d)

Figure 3: (Color online) $Q_{1,2}$ varies with dimensionless time $\omega t$ for different $\omega_{12}$, when $\delta=\Delta=0, T=10 \omega_{0}, T_{e}=0.2 \omega$, and $\lambda=0.1 \omega$ are chosen.

The squeezing vanishes rapidly when the phonon effect is enhanced which shows that the phonon effect is distinct in this field-QDM interaction model. Despite these factors, increasing the ratio of detuning $\omega_{12}$ to $\delta$ can weaken the squeezing. In addition, the initial mean phonon numbers can play an important role in the squeezing; the big number makes the squeezing behavior obscure. Compared with the atomic assembled counterpart, the QDM has its advantage that is more convenient for the experimental manipulation and this field-QDM model opens up the new possibility of studying the light squeezing and allows its use in tunable semiconductor quantum-dot system.

\section{Conflict of Interests}

The authors declare that there is no conflict of interests regarding the publication of this paper.

\section{Acknowledgments}

This work is supported by the National Natural Science Foundation of China under Grant nos. 11047101, 11105039, 11204274, 11205071, and 11264039 and by Jiangsu Provincial Natural Science Foundation under Grant no. BK2011462. 


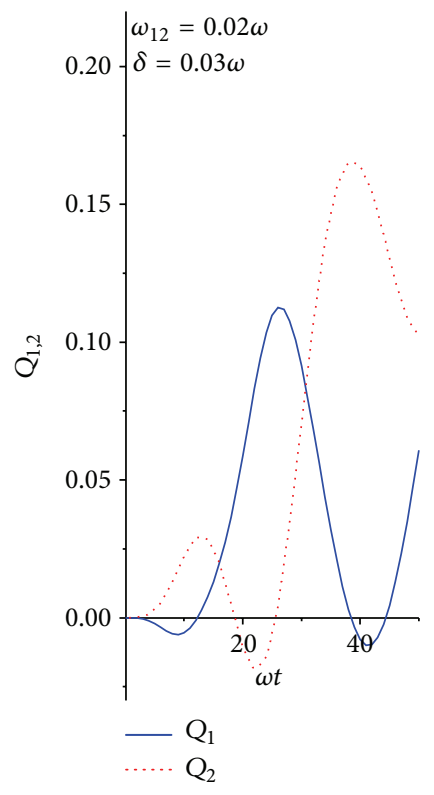

(a)

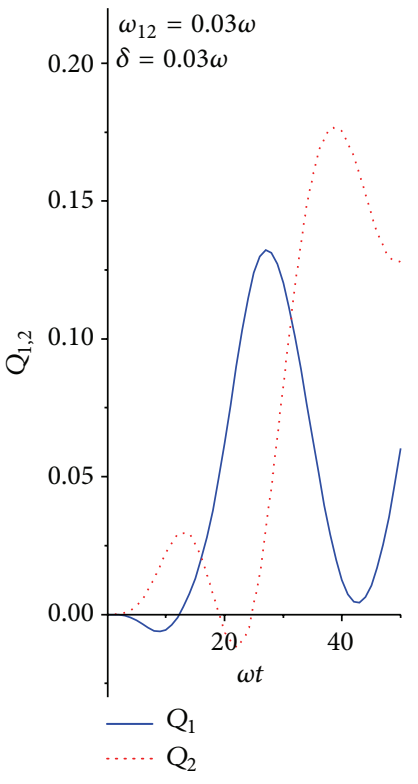

(b)

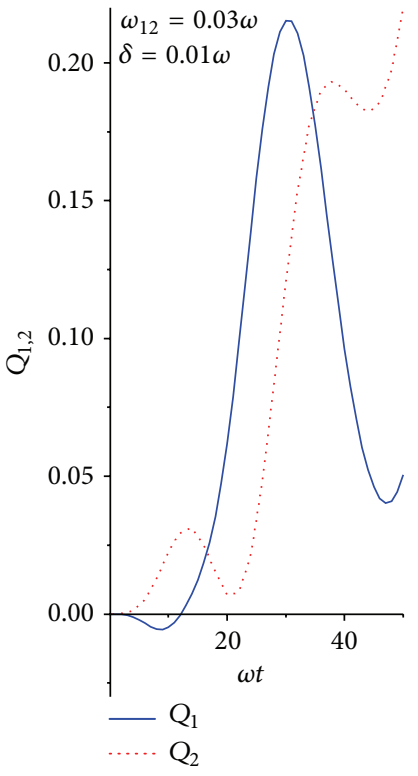

(c)

Figure 4: (Color online) $Q_{1,2}$ versus dimensionless time $\omega t$ for different detunings $\omega_{12}$ and $\delta$ with the same parameters chosen as previously.

\section{References}

[1] M. A. Reed, J. N. Randall, R. J. Aggarwal, R. J. Matyi, T. M. Moore, and A. E. Wetsel, "Observation of discrete electronic states in a zero-dimensional semiconductor nanostructure," Physical Review Letters, vol. 60, no. 6, pp. 535-537, 1988.

[2] E. A. Stinaff, M. Scheibner, A. S. Bracker et al., "Optical signatures of coupled quantum dots," Science, vol. 311, no. 5761, pp. 636-639, 2006.

[3] J. R. Kukliński and J. L. Madajczyk, "Strong squeezing in the Jaynes-Cummings model," Physical Review A, vol. 37, pp. 31753178, 1988.

[4] B. Rodríguez-Lara, H. Moya-Cessa, and A. B. Klimov, "Combining Jaynes-Cummings and anti-Jaynes-Cummings dynamics in a trapped-ion system driven by a laser," Physical Review A, vol. 71, no. 2, Article ID 023811, 2005.

[5] A. Kundu, "Quantum integrable multi-atom models that describe the interaction between radiation and matter with and without the rotating-wave approximation," Theoretical and Mathematical Physics, vol. 144, no. 1, pp. 975-984, 2005.

[6] V. Hussin and L. M. Nieto, "Ladder operators and coherent states for the Jaynes-Cummings model in the rotating-wave approximation," Journal of Mathematical Physics, vol. 46, no. 12, Article ID 122102, 21 pages, 2005.

[7] E. T. Jaynes and F. W. Cummings, "Comparison of quantum and semiclassical radiation theories with application to the beam maser," Proceedings of the IEEE, vol. 51, no. 1, pp. 89-109, 1963.

[8] B. W. Shore and P. L. Knight, "The Jaynes-Cummings model," Journal of Modern Optics, vol. 40, no. 7, pp. 1195-1238, 1993.

[9] D. F. Walls, "Squeezed states of light," Nature, vol. 306, no. 5939, pp. 141-146, 1983.

[10] C. M. Caves, K. S. Thorne, R. W. P. Drever, V. D. Sandberg, and M. Zimmermann, "On the measurement of a weak classical force coupled to a quantum-mechanical oscillator. I. Issues of principle," Reviews of Modern Physics, vol. 52, no. 2, pp. 341-392, 1980.
[11] H. Grote, K. Danzmann, K. L. Dooley, R. Schnabel, J. Slutsky, and H. Vahlbruch, "First long-term application of squeezed states of light in a gravitational-wave observatory," Physical Review Letters, vol. 110, no. 18, Article ID 181101, 2013.

[12] M. O. Scully and M. S. Zubairy, Quantum Optics, Cambridge University Press, Cambridge, UK, 1997.

[13] A. D. O'Connell, M. Hofheinz, M. Ansmann et al., "Quantum ground state and single-phonon control of a mechanical resonator," Nature, vol. 464, no. 7289, pp. 697-703, 2010.

[14] S. Yang, S. L. Zhang, X. B. Zou, S. W. Bi, and X. L. Lin, "Improving noiseless linear amplification for optical quantum communication with quadrature squeezing," Physical Review A, vol. 87, Article ID 024302, 2013.

[15] A. A. Clerk, F. Marquardt, and K. Jacobs, "Back-action evasion and squeezing of a mechanical resonator using a cavity detector," New Journal of Physics, vol. 10, Article ID 095010, 2008.

[16] J. M. Villas-Bôas, A. O. Govorov, and S. E. Ulloa, "Coherent control of tunneling in a quantum dot molecule," Physical Review B, vol. 69, Article ID 125342, 2004.

[17] C.-R. Du and K.-D. Zhu, "Phonon effect on two coupled quantum dots at finite temperature," Physics Letters A, vol. 372, no. 4, pp. 537-540, 2008.

[18] L. Fedichkin and A. Fedorov, "Error rate of a charge qubit coupled to an acoustic phonon reservoir," Physical Review A, vol. 69, no. 3, Article ID 032311, 2004.

[19] Z. Wu, K. Zhu, X. Yuan, Y. Jiang, and M. Yao, "Phonon effect on an optically driven quantum dots device," Physics Letters A, vol. 347, no. 4-6, pp. 251-254, 2005.

[20] K. Huang and A. Rhys, "Theory of light absorption and nonradiative transitions in F-centres," Proceedings of the Royal Society of London A, vol. 204, pp. 406-423, 1950.

[21] P. Meystre, E. Geneux, A. Quattropani, and A. Faist, "Longtime behaviour of a two-level system in interaction with an electromagnetic field," Il Nuovo Cimento B, vol. 25, no. 2, pp. 521-537, 1975. 
[22] T. von Foerster, "A comparison of quantum and semi-classical theories of the interaction between a two-level atom and the radiation field," Journal of Physics A: Mathematical and General, vol. 8, p. 95, 1975.

[23] H. Kamada, H. Gotoh, J. Temmyo, T. Takagahara, and H. Ando, "Exciton rabi oscillation in a single quantum dot," Physical Review Letters, vol. 87, no. 24, Article ID 246401, 2001.

[24] N. H. Bonadeo, J. Erland, D. Gammon, D. Park, D. S. Katzer, and D. G. Steel, "Coherent optical control of the quantum state of a single quantum dot," Science, vol. 282, no. 5393, pp. 1473-1476, 1998.

[25] C. Piermarocchi and L. J. Sham, "Control of exciton dynamics in nanodots for quantum operations," Physical Review Letters, vol. 87, no. 6, Article ID 067401, 2001.

[26] T. Calarco, A. Datta, P. Fedichey, E. Pazy, and P. Zoller, "Spinbased all-optical quantum computation with quantum dots: Understanding and suppressing decoherence," Physical Review A, vol. 68, no. 1, Article ID 012310, 2003.

[27] A. Tackeuchi, T. Kuroda, K. Mase, Y. Nakata, and N. Yokoyama, "Dynamics of carrier tunneling between vertically aligned double quantum dots," Physical Review B, vol. 62, no. 3, pp. 1568-1571, 2000.

[28] C. Emary and L. J. Sham, "Optically controlled logic gates for two spin qubits in vertically coupled quantum dots," Physical Review B, vol. 75, Article ID 125317, 2007. 

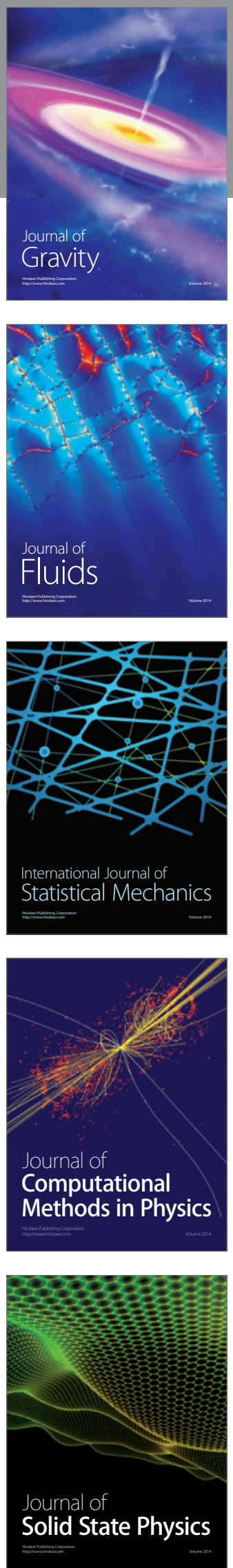

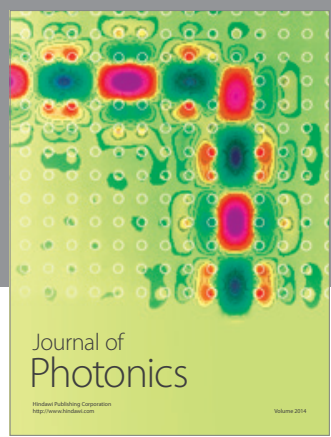

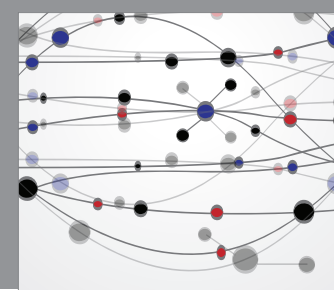

The Scientific World Journal

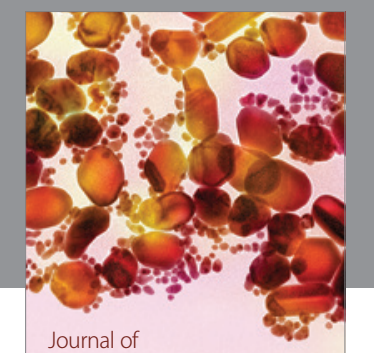

Soft Matter
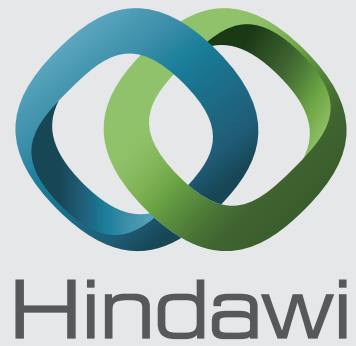

Submit your manuscripts at

http://www.hindawi.com
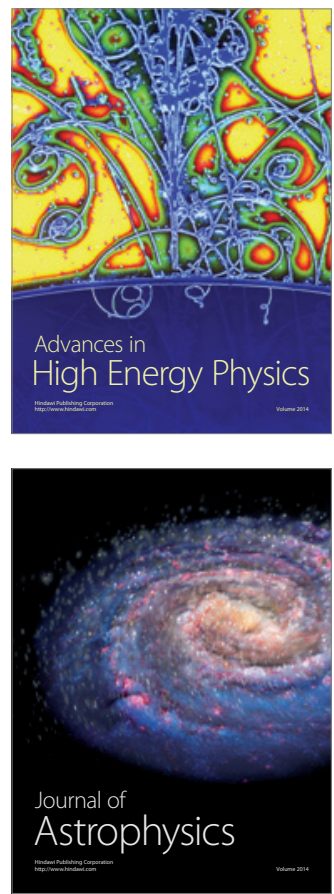
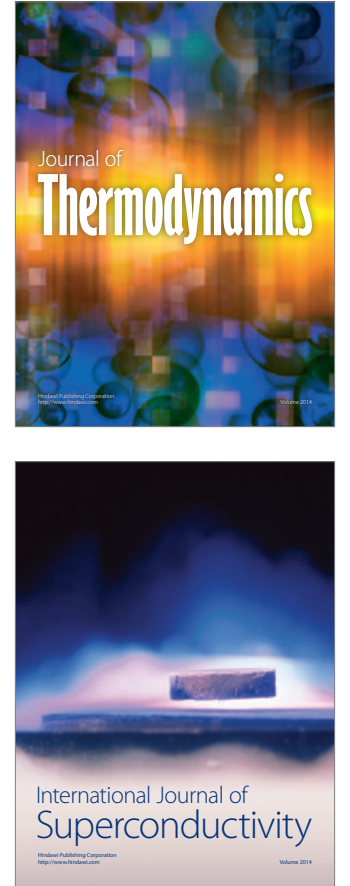
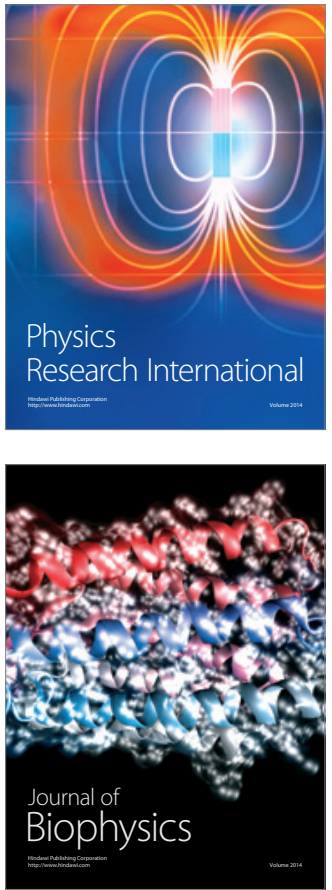
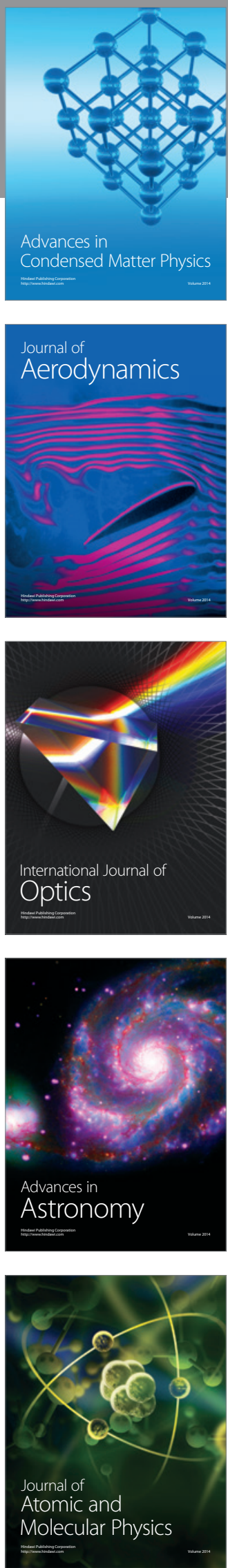